\title{
A Robust Inter-Domain DDS Gateway based on Token Passing for Large-Scale Cyber-Physical Systems
}

\author{
Wooyeob Lee*, Sungmoon Chung*, Moonwon Choi*, Sungryung Cho*, Inwhee Joe*, \\ Jeman Park**, Soohyung Lee**, Wontae Kim** \\ * Department of Electronics and Computer Engineering, Hanyang University, Korea \\ ** ETRI (Electronics and Telecommunications Research Institute), Korea \\ \{matias12, dear1115, c0322h, kyougt, iwjoe\}@hanyang.ac.kr, \{jeman, soohyung, wtkim\}@etri.re.kr
}

\begin{abstract}
In this paper, we propose the architecture of the Data Distribution Service Gateway (DDS GW) to interconnect different DDS domains for large-scale Cyber-Physical Systems (CPS).

We suggest the DDS GW service which runs on the DDS Middleware. It consists of four major components: Interface Module, Topic Manager, Routing Manager and Network Module.

We also suggest a basic algorithm for resolving the bottleneck problem caused by the exchanges of EDP messages.
\end{abstract}

Keywords - Cyber-Physical System, CPS Middleware, InterDomain, DDS Gateway

\section{INTRODUCTION}

Cyber Physical System (CPS) [1] is an interconnection among the heterogeneous computing devices providing the highly reliable real-time control of distributed physical systems through networks. To this purpose, it is necessary to develop the CPS Middleware for the efficient data exchanges in the CPS environment. In most researches, the term "CPS Middleware" is described as the middleware which is based on the data-centric manner and guarantees real-time performance. In this regard, many of them say that the Object Management Group (OMG)'s DDS [2] is the most suitable middleware for CPS.

For the inter-domain connection, OMG's DDS provides the Bridging mechanism among the DDS domains [3], but it is only for the domains which are connected to the same network switch. In this regard, it is essential to consider general interdomain networks, which are located far away, to achieve the complete CPS scenario.

Therefore, in this paper, we propose the architecture and the procedure of DDS GW for the interconnection among the CPS domains. In the following chapter, we introduce a brief description of DDS Middleware and its interconnection scheme. Chapter III contains the detail architecture of DDS GW and a Token Passing algorithm. In chapter IV, we evaluated and compared our Token passing algorithm to the simple GW which has no algorithm to resolve the bottleneck problem.

\section{OMG DDS}

DDS is a middleware which is standardized by OMG. It is based on the Publish and Subscribe messaging pattern which is fit for the large-scale data transmission and it has more realtime features compared to the Client-Server pattern.

Participants, the core member of DDS domain, communicate with each other in same Domain (or Data Space) by using the Endpoints.

\section{A. Discovery Procedure in OMG DDS}

As mentioned previously, The OMG DDS is based on the Publish and Subscribe messaging pattern. All active Participants periodically send the Participant Discovery Protocol (PDP) messages to each other. If the Participant receives the PDP message, it replies immediately with its PDP message. After the PDP process, they exchange the Endpoint Discovery Protocol (EDP) messages via unicast. The number of sent EDP messages from each Participant is equal to the number of Endpoints in each Participant. Once the EDP messages are exchanged, the Participants check the existence of the Matched Endpoint. If it exists, they perform the QoS negotiation and start to send the data.

However, there occur congestion problems if the number of Participants which cause a large number of PDP and EDP messages increases. And there are some methods to solve this problem [4].

\section{B. Inter-Domain Mechanism in OMG DDS}

The OMG DDS provides a Context-Aware Bridging scheme as the inter-domain mechanism [3].

As its name suggests, the Context-Aware Bridging is the bridging scheme which works only when the Domains are connected directly with same switch.

They added the Routing layer between the Pub/Sub layer and the Application layer. This new layer is included only in the DDS-IS node which works as the Bridge and therefore, DDS-IS node is connected to both domains. It receives all discovery messages from both domains, filters them and delivers them to opposite domains. 
As the Routing Layer manages the number of EDP messages by filtering them, the number of delivered EDP messages would be decreased. But, in the worst case, the DDS-IS will become a bottleneck. And it is not suitable for the CPS environment, since it does not provide the interconnection between the domains which are located far away. Therefore, we propose the Inter-Domain GW architecture to meet the needs for the interconnection among the distant domains.

\section{INTER-DOMAIN DDS GATEWAY}

\section{A. Architecture of DDS Gateway}

The DDS GW is an application which runs as a service on the DDS middleware. The proposed architecture is as follow.

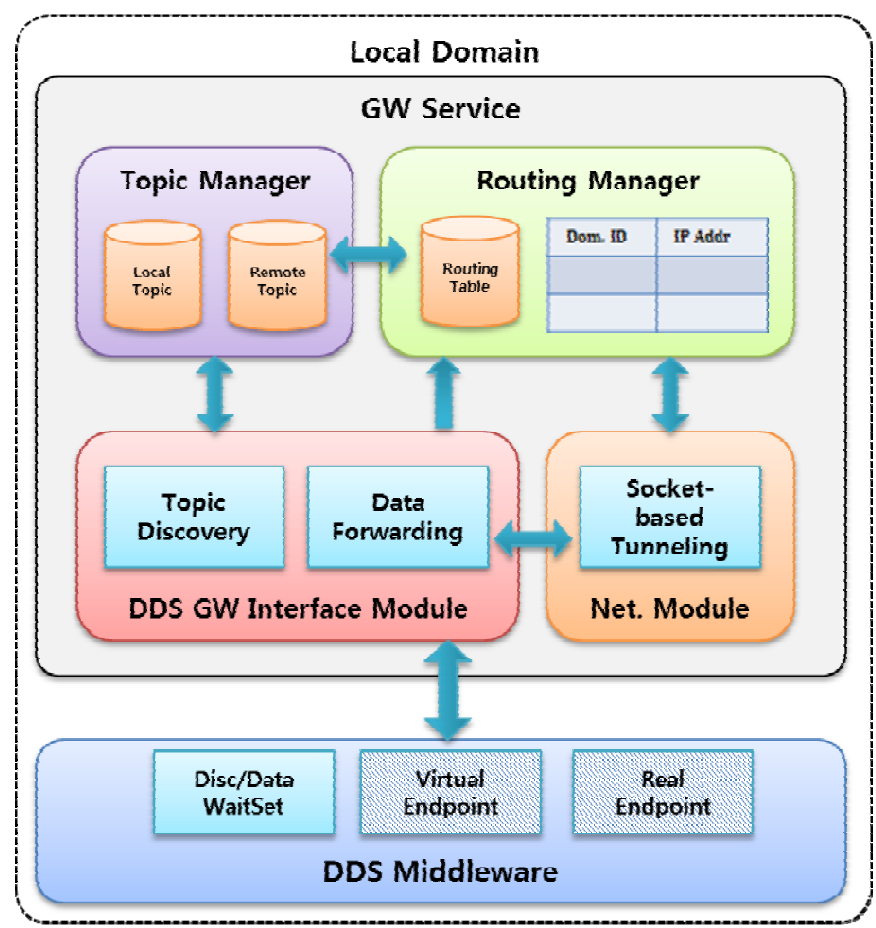

Figure 1. Overall Architecture

It is composed of four components.

1) DDS GW Interface Module (DDS GW IF Module): It is the interface module between the DDS middleware and the $\mathrm{GW}$ service. It responsible for handling the events occurred from the DDS or sent from other GW and there are two main features of IF module: the Topic Discovery and the Data Forwarding.

The Topic Discovery sub-module detects the incoming of the EDP messages from the DDS middleware. For this, we use the WaiSet of the DDS as a trigger. It also handles the EDP messages received from other GWs.

The Data Forwarding forwards the data to other GW or to the DDS Middleware. It also uses the Waitset to trigger the Data from the DDS middleware.
2) Topic Manager: The Topic Manager manages the Topic (EDP) Database. The Topics from the DDS middleware are stored in Local Topic DB and the Topics from other GWs are stored in Remote Topic DB adding the Tag which indicates the source GW of the Topic.

3) Routing Manager: The Routing Manager provides the routing table to map the address of other GWs. It manages the Domain ID, which is considered as the ID of GW, and the IP address of the corresponding GW.

4) Network Module: This Module takes charge of all communications among the GWs. It establishes two TCP connections per each of all active GWs: One for Upload (Sending) and another for Download (Receiving). The role of this module is just sending or receiving the messages to/from other GWs.

\section{B. Procedure of DDS GW Service}

The procedure of propose architecture is divided in three stages: Gateway Initialization, Discovery Process and Data Transmission.

1) Gateway Initialization: First, the GW Service daemon creates all modules as the initialization of the DDS Gateway. Once all modules are created, it loads the XML file which contains the initial parameters and information about other GWs. Then, it establishes two TCP connections per all active GWs. After the establishment, it requests the creation of DDS Entity and starts to operate as DDS GW. In this paper, we assume that the information stored in XML file is absolutely the same as the real information of the connected GW.

2) Discovery Process: Since, in our proposed architecture, the Participant which belongs with each of GWs acts as a proxy of all Participants of other Domains, the transmission of PDP messages are not required and it just sends the EDP messages. The delivery of EDP messages is divided in two processes. One is for the Local Topic transmission toward other GWs and another process is for receiving the Remote Topic.

When the IF module receives the EDP message sent from DDS Entity, it forwards the EDP message to the Topic Manager and the Topic Manager stores it in Local Topic DB. Then, the IF request to send the EDP message to all connected GWs. If the GW receives the EDP message, it stores the received EDP message in the Remote Topic DB. Then, it forwards the message to the DDS Entity. Since the DDS Entity recognizes the forwarded EDP message as new data, DDS Entity creates a new Endpoint for the corresponding EDP message and sends it to all discovered Participants in the same Domain.

However, as the number of Remote Topics is very large, there occurs a huge lack of resources by creating the Endpoints for all Remote Topics. For this reason, to reduce the waste of resources, firstly we add an unallocated Endpoint, or Virtual Endpoint (VEP) to the Endpoint List. When the Matched Endpoint is detected, DDS allocates the resource for the corresponding VEP and the VEP becomes Real Endpoint. 
3) Data Transmission: The data transmission process is quite simple than that of discovery process. When the data is received, the Data Forwarding sub-module identifies the destination by using the state information in Data Forwarding sub-module and Remote Topic DB.

\section{Token Passing Scheduling Algorithm}

In this section, we explain our proposed scheduling algorithm based on token passing. We assume that all gateways have their order of priority. Thus, the gateways can transmit their EDPs in a sequence at their own order.

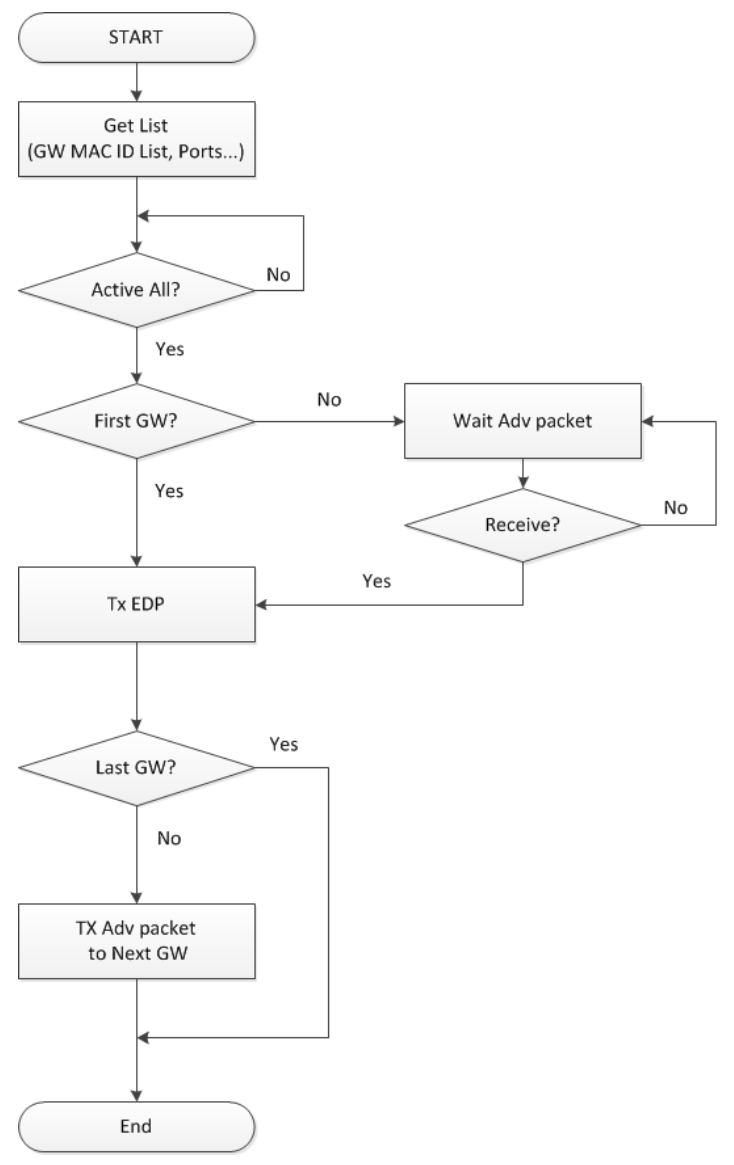

Figure 2. Proposed scheduling algorithm

Step1. Start scheduling algorithm.

Step2. Get the GW list from routing manager. At this moment, we get the list of all possible candidate GWs.

Step3. Check whether all gateways are active or not. If not, check again. This process is to wait the late join in the initial state.

Step4. Check whether it is first gateway or not. If it is, goes Step6 to run the token creation process. If it isn't go Step5 to wait the token.

Step5. Wait ADV(advertise) message. If it receives the message, go Step6. If not, check again.

Step6. Transmit EDP to the other gateway.

Step7. Check whether it is last gateway or not. If it is last gateway, go Step9.

Step8. Transmit ADV to next gateway.
Step9. End scheduling algorithm.

\section{IV.PERFORMANCE EVALUATION}

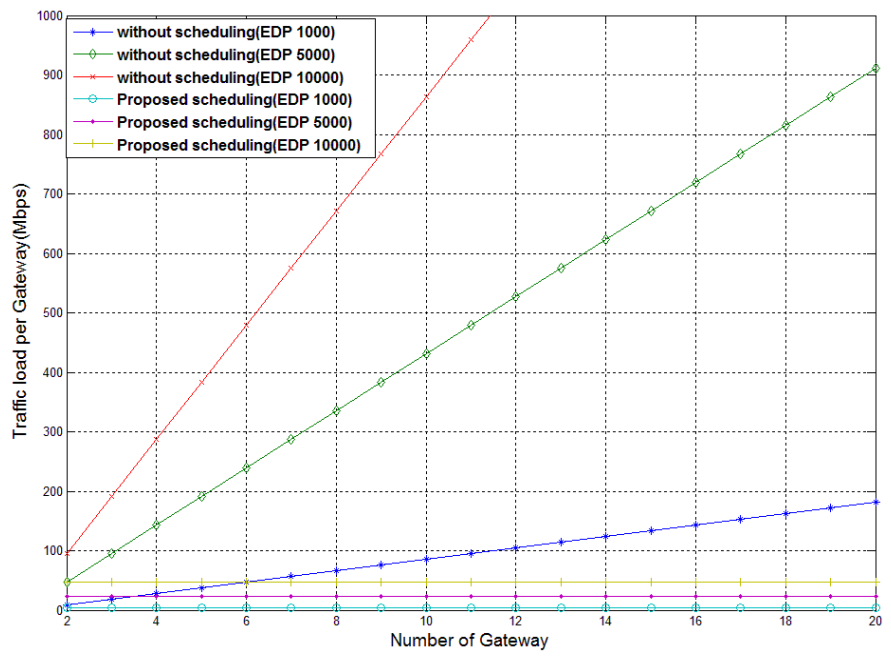

Figure 3. Traffic load performance per gateway

Our proposed scheduling scheme is based on token passing. In the basic architecture, there would be a number of transmitting gateways and the Bottleneck problem may occur. In the proposed algorithm, there is only one transmitting gateway at a time because, in our proposed algorithm, the only one gateway which has the ADV can transmit EDP messages. Therefore, the traffic load per gateway is decreased in a single moment. As shown in fig 3, our proposed scheduling algorithm is more outperformed than the architecture without scheduling algorithm. The gap in traffic load performance between two cases will get bigger as the number of deployed gateways is increased.

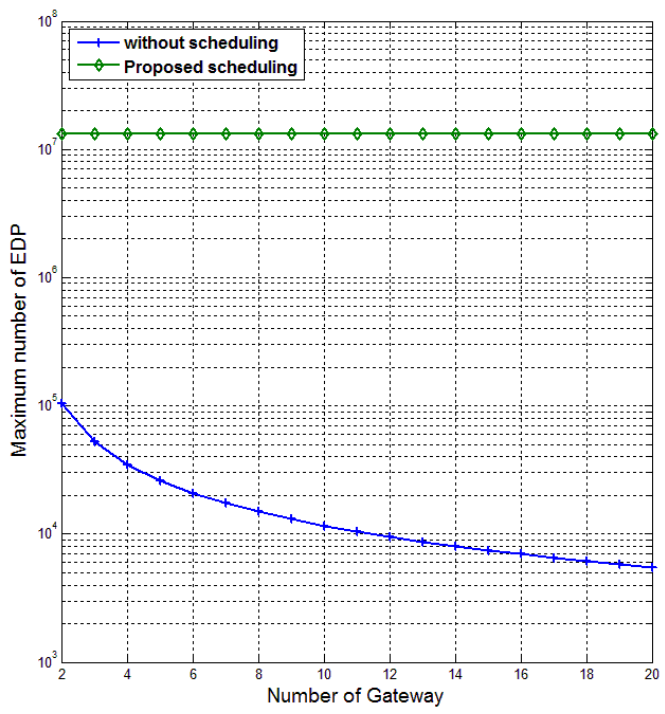

Figure 4. Maximum number of EDPs per gateway 
As shown in fig 4, the maximum number of EDP messages of the proposed algorithm is always the same regardless of the number of gateways. However, without the scheduling algorithm, the maximum number of transmitting gateways is decreased sharply as the number of gateways increases.

\section{CONClusions}

In this paper, we proposed the architecture and the scheduling algorithm for DDS GW to provide a robust interconnection among the CPS domains. We defined the basic architecture of DDS GW and suggested solution for the Bottleneck problem by using the Token Passing. We demonstrated that our architecture and algorithm is suitable for the CPS systems.

\section{ACKNOWLEDGMENT}

This work was supported by the IT R\&D Program of MSIP/KEIT [10035708, "The Development of CPS (CyberPhysical Systems) Core Technologies for High Confidential Autonomic Control Software"].

\section{REFERENCES}

[1] http://cyberphysicalsystems.org/

[2] OMG, Data Distribution Service for Real-time Systems Specification Ver 1.2, 2007.

[3] J. M. Lopez-Vega, J. Povedano-Molina, G. Pardo-Castellote, J. M. Lopez-Soler, "A content-aware bridging service for publish/subscribe environments"., The Journal of Systems and Software, Vol. 86, pp. 108-124, 2012.

[4] J. Park, W. Lee, S. Chung, I. Joe, W. Kim "A fast and scalable service discovery protocol for CPS-based warships", Proceedings of IEEE International Conference on Innovative Computing and Communication (CICC2011), Vol. 2, pp. 335-338, 2011.

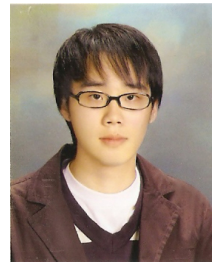

Woo-Yeob Lee received his B.S. and M.E. degree in Computer Science \& Engineering from Hanyang University, Seoul, Korea in 2009. Currently he has done his $\mathrm{PhD}$ in the year 2009 from Hanyang University, His current research interests include Cyber Physical System, Cloud Computing, Cognitive Radio, D2D and Beyond 5G.

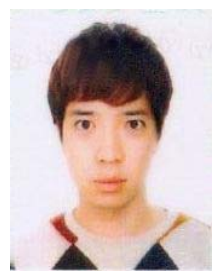

Sungmoon Chung did his B.S. in Division of Computer Science \& Engineering and MS in Department of Electronics \& Computer Engineering from Hanyang University, HengDangDong, Seoul. Currently he has done his $\mathrm{PhD}$ in the year 2009 from Hanyang University, Haengdang-dong, Seoul from the Department of electronics computer engineering. His research interest includes computer networking such as wireless sensor networks, 4G/5G celluar networks and CPS(Cyber Physical System).

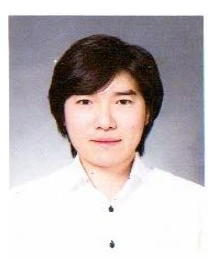

Moonwon Choi received his M.S. degrees in Department of Computer Engineering from Hanyang University,
HengDangDong, Seoul, Korea. Since 2011, he has done his Ph.D from Hanyang University, His current research interests include wireless sensor/ad hoc networks, convergence, A.I and Cyber Physical System.

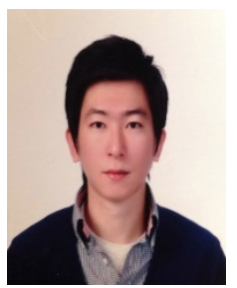

Sungryung Cho received his B.S. degrees in department of Electrial engineering \& Computer science from Kookmin University, Seoul, Korea in 2012. Since 2013, he has done his M.S. degree in Computer \& Software from Hanyang University, Seoul, Korea. His current research interests include wireless sensor network, and CPS(Cyber Physical System).

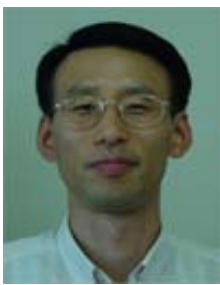

Inwhee Joe received his B.S. and M.S. degrees in Electronics Engineering from Hanyang University, Seoul, Korea, and his Ph.D. degree in Electrical and Computer Engineering from Georgia Institute of Technology, Atlanta, GA in 1998. Since 2002, he has been a faculty member in the Division of Computer Science \&Engineering at Hanyang University, Seoul, Korea. His current research interests include mobile internet, cellular system and PCS, wireless ATM ,mobile adhoc networks, multimedia networking, performance evaluation.

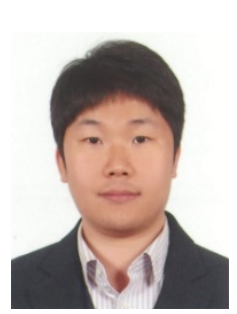

Jeman Park received his B.S. and M.S degrees in Electronics Engineering from Hanyang University, Seoul, Korea. Since 2012, he has been a faculty member of CPS Research Team at ETRI, Daejeon, Korea. His current research interests include Future internet, wireless sensor/ad hoc networks, 3G/4G cellular systems and Cyber Physical System.

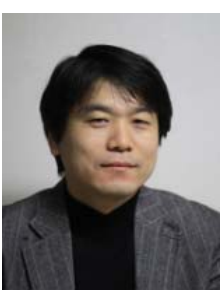

SooHyung Lee received his Ph.D. in Computer Engineering from ChungNam National University in 2012 and BS, MS degrees from the department of Electronic Engineering, Hanyang University, Korea in 1991 and 1993 respectively. In August 1993, he joined the network design laboratory of DACOM Corporation. Since October 2000, he has been a Principal Member of Engineering Staff, CPS research team, Electronics and Telecommunications Research Institute (ETRI), Korea. His research interests include IT converging system, distributed communication, network security.

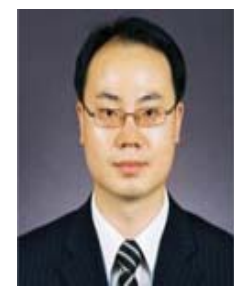

Won-Tae Kim received his $\mathrm{BE}, \mathrm{ME}$, and $\mathrm{PhD}$ degrees in Electronic Engineering from Hanyang University, Seoul, Korea in 1994, 1996, and 2000, respectively. He was CTO of Rostic Technologies, a venture company developing advanced mobile technologies during 2001-2005. In March 2005 he joined Embedded Software Development Division at ETRI. His major interests are: advanced mobile networks, mobile middleware and CPS-based computing. 